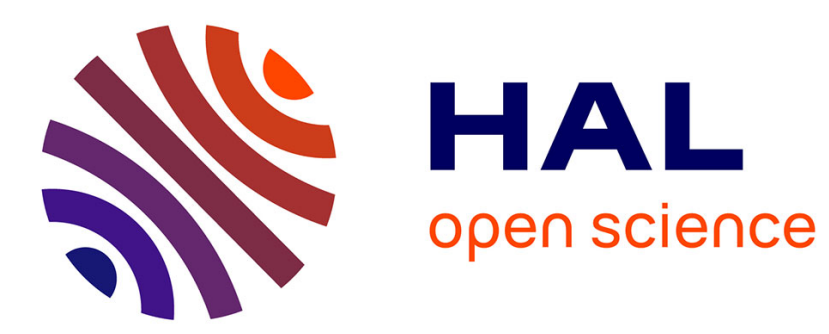

\title{
Attitude Estimation for Posture Detection in eHealth Services
}

\author{
Aida Makni, Grégoire Lefebvre
}

\section{To cite this version:}

Aida Makni, Grégoire Lefebvre. Attitude Estimation for Posture Detection in eHealth Services. 31st IEEE Computer-Based Medical Systems, Jun 2018, Karlstad, Sweden. hal-01825808

\section{HAL Id: hal-01825808 \\ https://hal.science/hal-01825808}

Submitted on 28 Jun 2018

HAL is a multi-disciplinary open access archive for the deposit and dissemination of scientific research documents, whether they are published or not. The documents may come from teaching and research institutions in France or abroad, or from public or private research centers.
L'archive ouverte pluridisciplinaire HAL, est destinée au dépôt et à la diffusion de documents scientifiques de niveau recherche, publiés ou non, émanant des établissements d'enseignement et de recherche français ou étrangers, des laboratoires publics ou privés. 


\section{Attitude Estimation for Posture Detection in eHealth Services}

\author{
Aida Makni \\ Orange Labs \\ $R \& D$ \\ Meylan, France \\ aidamakni@gmail.com
}

\author{
Grégoire Lefebvre \\ Orange Labs \\ $R \& D$ \\ Meylan, France \\ gregoire.lefebvre@orange.com
}

\begin{abstract}
We investigate the influence of two attitude estimation methods for human posture detection. In the context of ADL (i.e. Activities of Daily Living) we analyze inertial sensors to reveal uncomfortable situations. Quantifying postures such as standing up, walking, lying down or sitting may feature people autonomy and well being. We report comparisons between two main attitude estimation strategies. Our experimental protocol uses a precise ground truth obtained from two annotators. The dataset involves 9 participants and provides 50 various data sequences. We discuss the obtained promising results, analyze advantages and limits when using attitude estimation in this context.
\end{abstract}

Keywords-Inertial Measurement Unit; Attitude Estimation; Posture Detection; eHealth; Actigraphy.

\section{INTRODUCTION}

Nowadays, eHeath services based on inertial sensors increasingly rely on methods for detecting human postures and actions. Human action recognition based on inertial sensors has been an active area of research due to its success in robotics [13], human computer interaction [6], guidance [7], etc. Actions may be viewed as sequences of static and dynamic postures during the time. Nevertheless, human posture detection remains challenging, in particular for eHealth applications [2]. Indeed, posture analysis in Activities of Daily Living (ADL) is crucial because it may reveal a disease, a loss of autonomy, difficult working conditions, etc. In this case, device attitude estimation (i.e. device orientation with respect to Earth's local frame [18]) is fundamental.

Modern IMUs (Inertial Measurement Units) embed accelerometer, gyroscope, and magnetometer sensors which enable methods for attitude estimation. These methods investigate various domains such as: aerospace [8], unmanned aerial vehicles [4], indoor positioning [15], etc. Moreover, the particular context of smartphones carried by people in their everyday lives brings new challenges for detecting their postures. If people let their phone on a pocket, upsidedown or in their backpack, attitude estimation is relevant to transform input data in a reference orthonormal coordinate system such as a torso relative one. In a torso linked referential, postures as standing up or lying down are indeed easier to detect. External accelerations, gyroscope variations and magnetic perturbations need to be controlled when attitude estimation is realized to prevent additional issues.

Our contributions are the investigation of a posture detection system precision in the context of everyday life scenarios. We consider classical postures (such as lying down, sitting, standing up, walking, etc.) with various human behaviors in an indoor environment. We then analyze, compare and evaluate two state-of-the-art methods for attitude estimation. One originality is an indirect evaluation of attitude estimation methods with posture detection error quantification in regards to a human-made ground truth. The optimal automatic system should be as smart as human perception of postures.

The paper is organized as follows: the next section briefly reviews the state of the art of posture detection systems and models. In section 3, we review two classical attitude estimation methods. In section 4, we describe our experimental protocol and results are presented. Finally, Section 5 summarizes our contributions and concludes the paper.

\section{Human Posture Detection}

Human posture detection based on IMU data may be described as challenging sequential pattern recognition. The main objective is to determine as precise as possible starting and ending timestamp of a labeled posture. Classically, as in [3], postures are defined as a hierarchical representation from a root posture as standing up to more precise situations as standing with right arm up or lying down with curled up legs on right side (see Fig. 1). Generally, automatic posture detection systems use 3 main strategies based on: heuristics, statistical inference or machine learning.

In the first strategy, heuristics from IMU data are built to detect postures. For instances, in [20], the authors develop a sleep posture estimator using 3-axis accelerometer data in order to prevent Obstructive Sleep Apnea Syndrome (OSAS). Likewise, when a device is worn on the torso such as a necklace, and if the $z$ accelerometer axis is collinear to the gravity force, some rules may be provided. For example, the detection of the standing up position is when $z$ accelerometer data are around $g$ and $x$ accelerometer are around 0 . In the same way, the detection of the lying down position is when $z$ accelerometer data are around 0 and $x$ 


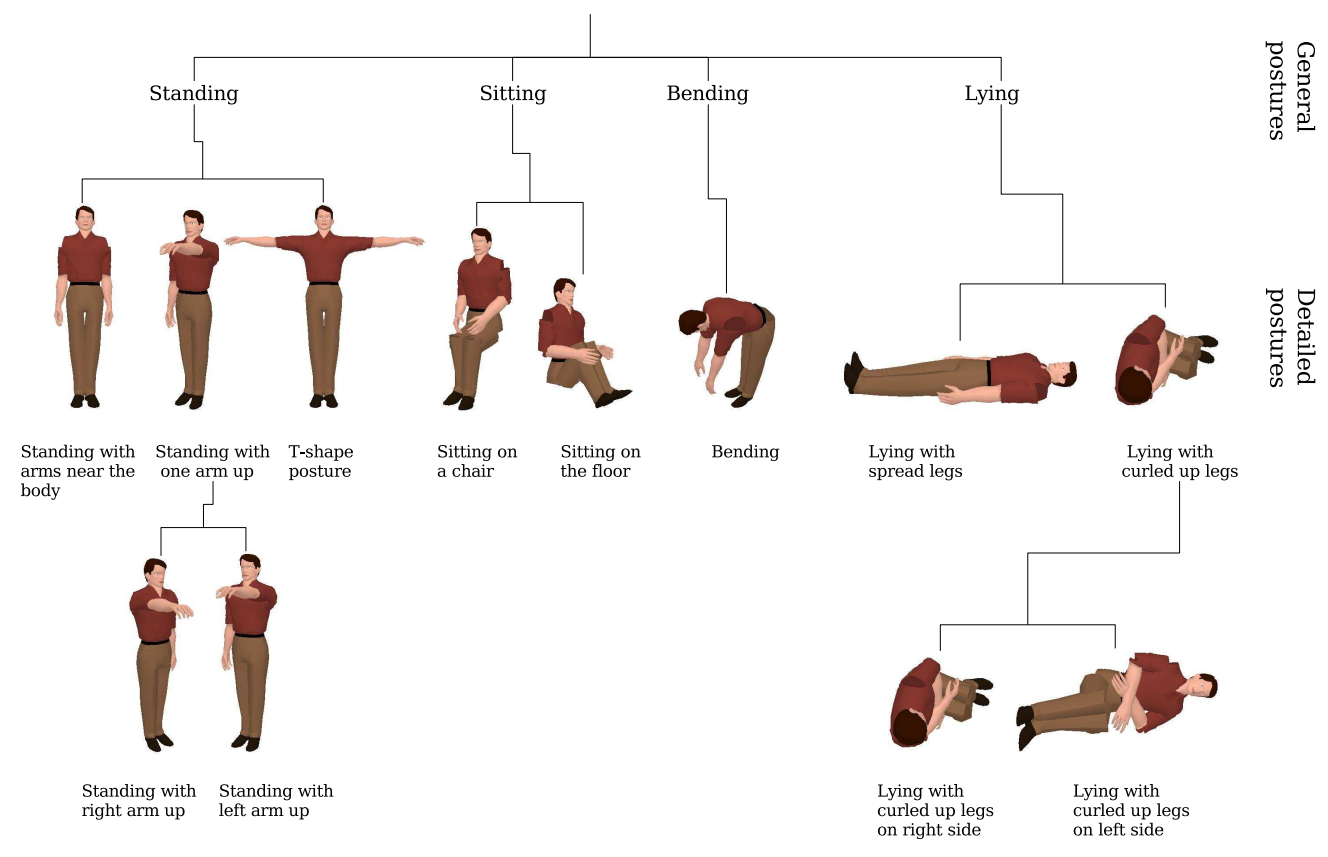

Figure 1: Hierarchical representation of human postures (extracted from [3]).

accelerometer are around $g$. Of course, threshold parameters should be fixed in advance, which is a real drawback for uncontrolled real life situations.

The second strategy suggests modeling the temporal structure of postures, with Hidden Markov Models (HMM). For instance, hand gestures in [14], which may be viewed as body postures, are modeled class by class from finite samples and the detection process consists of maximizing the likelihood of each class model outputs from input data.

The third method is to learn specialized classifiers (e.g. Support Vector Machine (SVM), Neural Networks (NN), etc.), which detect specific human posture from IMU data. In [12], Nelson et al. study how to maintain a healthy living for patients with Mild Cognitive Impairment (MCI). Their system based on IMU data models people activities with SVM based on their posture feature vectors.

In this study, we investigate the coupling between a posture detection method and two attitude estimators. Consequently, we build a posture detection algorithms based on the first strategy with heuristics in order to evaluate the attitude estimator added values. Fig. 2 shows our design. First, classical signal processing methods are applied to filter and denoise raw IMU data (i.e. a Butterworth filter with a Cutoff frequency of 0.01). Then, an attitude estimator is computed (see Section III) in order to calibrate data in a torso based referential. Following the next steps, rules are combined to classify IMU data to 5 human postures: walking, bending, lying down, standing up and sitting. From accelerometer data, dynamic versus static phase is obtained by thresholding data variation on a sliding window. For dynamic phases, we label temporal segments as walking and bending in regard to a step detector. Here, successive peaks on accelerometer energies with a frequency between $0.6 \mathrm{~Hz}$ and $2.5 \mathrm{~Hz}$ define a walking step. For static phases, we test first a lying and in second a standing pattern detectors based on samples revealing these situations. Finally, if no label is assigned after the lying and standing detectors, then the remain segments are classified as sitting postures. The schema relies then on a device attitude estimator, which may strongly influence the automatic posture detection accuracy.

\section{Device Attitude Estimation}

\section{A. Background}

Attitude estimation using IMU data is an important task in order to give an accurate posture detection for ADL monitoring. The 3-dimensional attitude of a rigid body is determined as the axes orientation of the body frame $B\left(X_{B}, Y_{B}, Z_{B}\right)$ with respect to a local Earth-fixed frame $N\left(X_{N}, Y_{N}, Z_{N}\right)$. It can be generally represented in 3 principal forms: Euler angles, quaternion, or Direction Cosine Matrix (DCM). The quaternion representation offers a linear formulation of the attitude dynamics in contrary to the other representations. In this paper, the unit norm quaternion is used to represent the attitude. It is defined as:

$$
q=\left[\begin{array}{ll}
q_{0} & \vec{q}^{T}
\end{array}\right]^{T}=\left[\begin{array}{llll}
q_{0} & q_{1} & q_{2} & q_{3}
\end{array}\right]^{T} \in \mathbb{R}^{4},
$$

where $q_{0}$ and $\vec{q}$ are the scalar and the vector parts of the quaternion, respectively. 


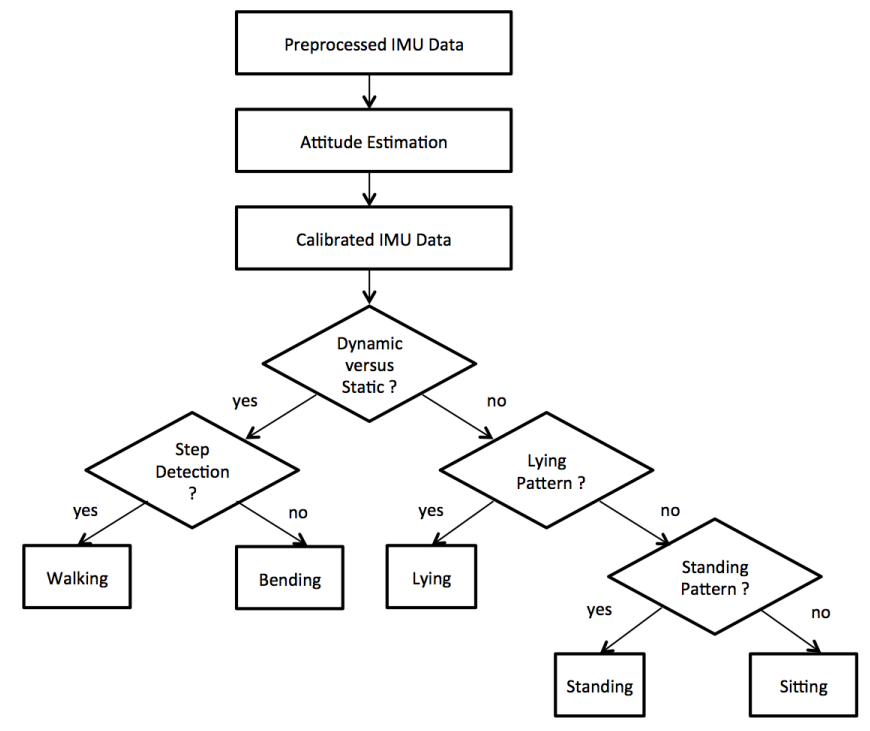

Figure 2: Posture Detection Automate with classic heuristics.

For IMU sensors, we consider a triad of sensors consisting of a gyroscope measuring the angular velocity, an accelerometer measuring the sum of the gravity and the device external acceleration and a magnetometer measuring the magnetic field. The classical kinematic equation to describe the variation of the attitude in term of quaternion is defined as follows [16]:

$$
\dot{q}=\frac{1}{2} q \otimes \bar{\omega},
$$

where $\bar{\omega}=\left[\begin{array}{ll}0 & \omega^{T}\end{array}\right]^{T}$ is the quaternion representation of the angular velocity $\omega=\left[\begin{array}{lll}\omega_{x} & \omega_{y} & \omega_{z}\end{array}\right]^{T}$ expressed in $B$.

The literature proposes different methods for attitude estimation [5], [9]-[11], [17]. In the following, we report the two different methods used in this study, exploiting firstly complementary filters [9] and secondly Kalman filters [10].

\section{B. Complementary Filters $(\mathrm{CF})$}

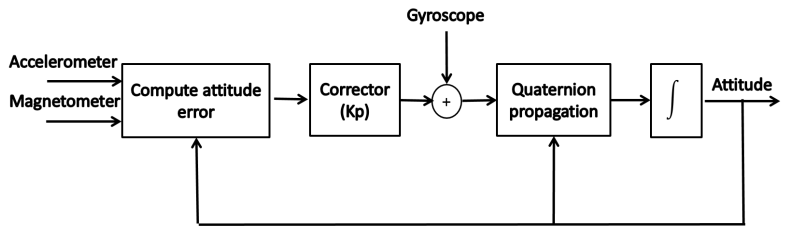

Figure 3: CF block diagram algorithm [9].

In the study of Mahony et al. [9], attitude estimation is performed from low cost IMU sensors. The input data is then very noisy with sampling period variations. In order to resolve these issues, the authors write the filtering problem as deterministic observers, linked on the Special Orthogonal group $\mathrm{SO}(3)$, driven by reconstructed attitude and angular velocity measurements. This method leads to determine two non-linear observers on $\mathrm{SO}(3)$, termed the direct $\mathrm{CF}$ and passive CF.

As described in the Fig. 3, the main idea is to calculate the error by cross-multiplying measured and estimated vectors. Then a correction step is used to correct the measured angular velocity. Again the quaternion propagation is integrated to obtain an estimate of the orientation.

The direct CF proposes non-linear observers using quaternion representations. The passive $\mathrm{CF}$ is derived from the reconstructed attitude based on the gyroscope measurements, avoiding coupling the constructed attitude error with the predictive velocity update. Additionally, the authors propose a formulation of the passive $\mathrm{CF}$ into an explicit $\mathrm{CF}$ from gravitational and magnetic field measurements that do not require online algebraic attitude reconstruction, which is a weakness for embedded systems. In this method, the attitude detector accuracy depends mainly on the $K_{p}$ gain parameter. Their experimental results appear very challenging on a robot and an unmanned aerial vehicle.

\section{Quaternion Adaptive Kalman Filters ( $q-A K F)$}

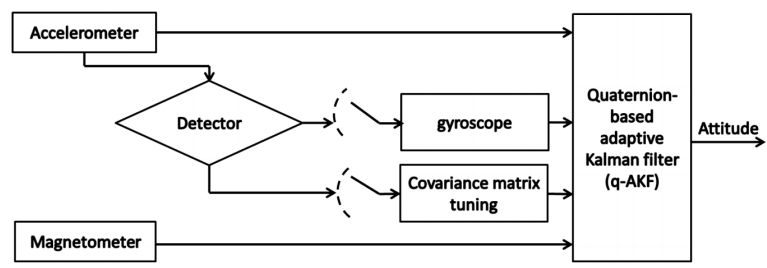

Figure 4: q-AKF block diagram extracted from [10].

Likewise, Makni et al. [10] consider the challenge of rigid object attitude estimation under external acceleration using IMU data. Their study focuses on attitude estimation during dynamic phases. Consequently, for compensating the external accelerations, a quaternion-based Adaptive Kalman filter (q-AKF) is designed (see Fig. 4).

First, a detector decides if the object is in a static or dynamic phase. Then, the external acceleration covariance matrix is estimated to feature the filter gain. The second challenge tacked in this study is the energy consumption issue when using a gyroscope. For preserving IMU battery life, the authors design a switching policy in order to exploit gyroscope data only when it is useful. The efficiency of the two contributions shows challenging results and the attitude detector accuracy depends mainly on the $P$ parameter, the initial value of the covariance matrix corresponding to the estimation error.

\section{EXPERIMENTS}

\section{A. Dataset}

1) Proposed scenario: The objective of the scenario in [1] is to collect inertial data of many participants in a reallife scenario (cf. Fig. 5). As activities of daily living are 


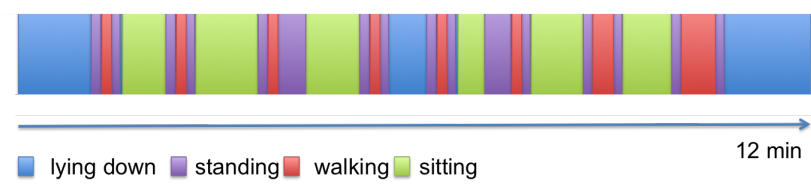

Figure 5: One sample posture sequence to be performed.

composed of 4 main postures (e.g. standing up, sitting, lying down, walking), the participants were asked to follow a particular protocol for about 12 minutes. Each participant performs a specific posture sequence in a particular order as follows: (standing up - walking - standing up - sitting). This sequence is repeated 6 times and may be preceded by a lying down posture or followed by a (standing up - walking - standing up - lying down) sequence. Each data sequence represents then human behaviors similar to an everyday life, but condensed in 12 minutes.

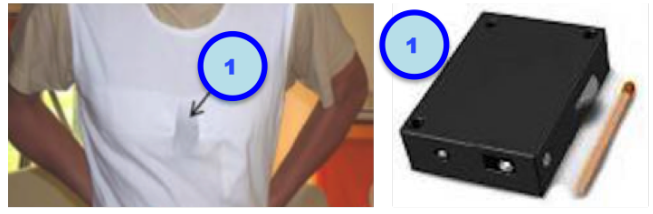

Figure 6: SK6 device.

2) Protocols: 9 participants (5 males and 4 females) were recruited to participate in the experiment [1]. The study was limited to French native participants in order to have homogeneous behavioral patterns and a standard annotation protocol. Ages are ranged from 22 to 55 years old with an average of 38 . Participants had different functions: interns, developers, PhD students, researchers, ergonomists and project managers.

A device, named SK6 [19], as shown in Fig. 6, was fixed on the participant and collect inertial data. 3D accelerometer, 3D gyroscope, 3D magnetometer data are sampled every 100 $\mathrm{ms}$, as shown in Fig. 7. The mean duration of each sequence is about 7643 samples (i.e. 13 minutes and 14 seconds long).

3) Data Annotation: One independent annotator segments manually each posture on the posture sequences in regards to the scenario, shown in Fig. 5. A second annotator validates the provided labels. This process was used for all inertial features in order to establish our ground truth (GT). The 4 main postures (i.e. lying down, sitting, standing up, walking) are labeled and also a bending label is added to mark the transition postures between lying down and sitting or standing up situations. These particular transition postures are indeed more fuzzy situations. This experiment design was thought to handle a list of classical postures known to largely contribute on activities of daily living. Finally, each sequence presents then around 35 annotated segments.
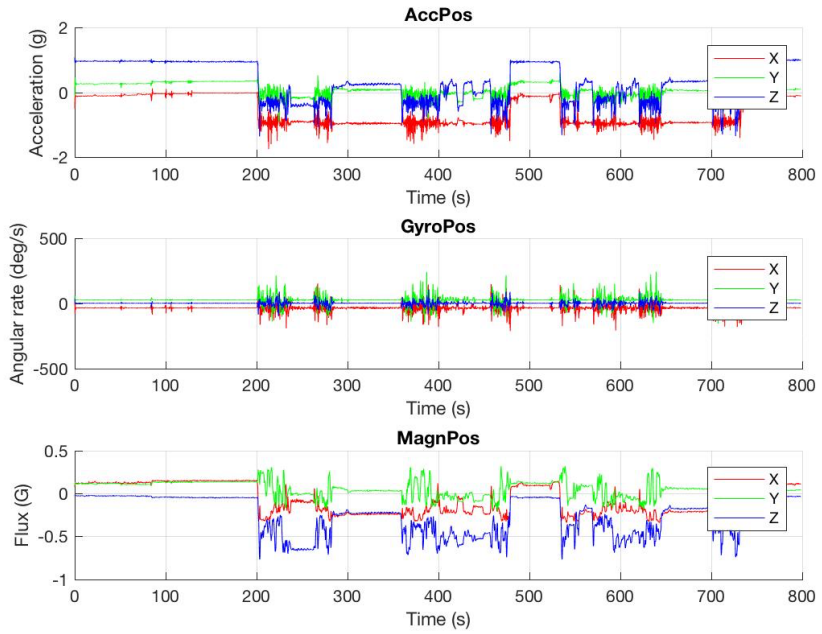

Figure 7: Accelerometer, gyroscope and magnetometer data for one participant during one IMU data acquisition.
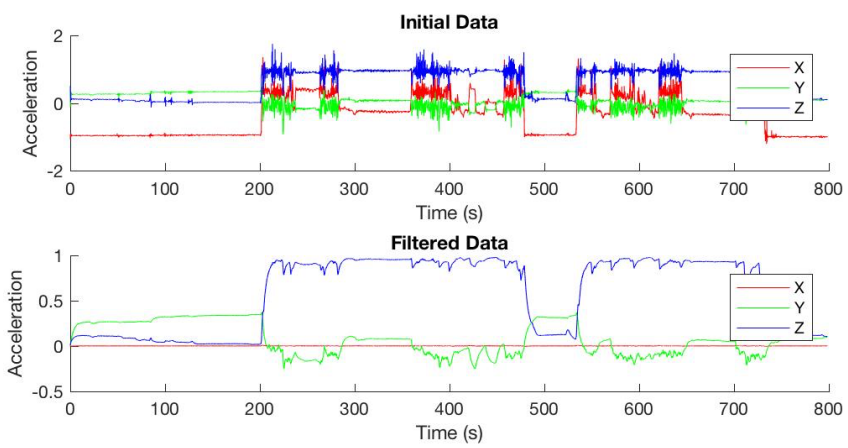

Figure 8: Raw and filtered accelerometer data for one participant.

\section{B. Results}

Fig. 8 presents raw data of one session and the filtered data, obtained before the attitude estimation phase, used for posture detection. We observe that the low-pass filter on the $z$-axis data provides a smoother signal, denoising then some interferences. The posture detector is then facilitated when automatically labeling walking or standing up signal segments. Likewise, signal processing on $y$-axis reveals clearly situations when participants are lying down (i.e. in the beginning of the scenario and around 500 seconds. The two resulting postures, namely sitting and bending are then deduced from the 3 previous ones, as described in Fig. 2.

Each labeled segments build finally a specific APD (Automatic Posture Detector-based) posturogram that is compared to the specific GT (Ground Truth) posturogram for each participant. Fig. 9 shows two posturograms for one participant and the superposition of the two previous ones.

It is then notable that on this user session, the automatic posture detector performs well, underlying the ground truth. 

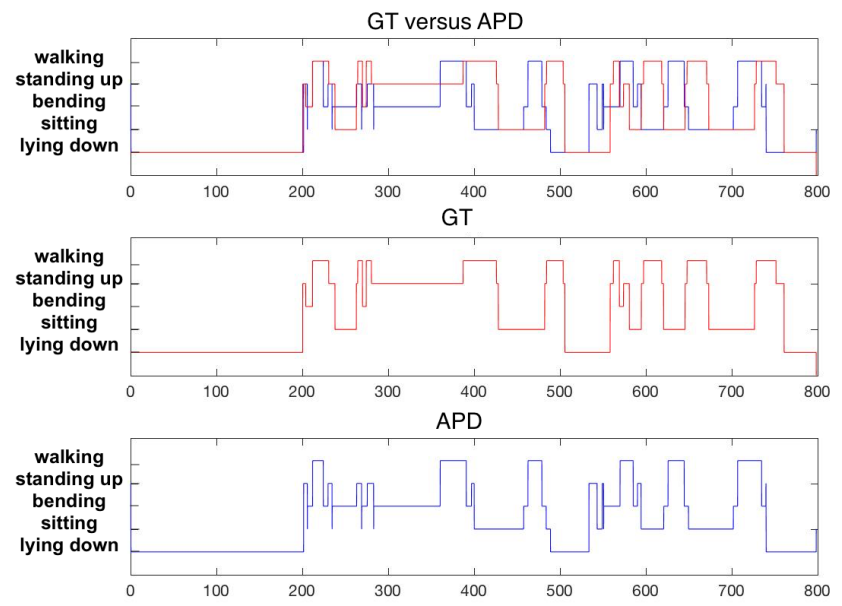

Figure 9: Posturogram alignments between the Ground Truth (GT) and the Automatic Posture Detection (APD).

Some errors appears when distinguishing standing up and bending postures. These two postures are quite similar in real life, and these errors are due to some standing detector imprecisions. Sometimes, some errors show light delays when detecting postures. These time variations may be caused by some issues to determine precisely starting times of a new posture. Again, bending postures are less marked situations and consequently more difficult to detect because they often result from transfer phases between two marked situations (i.e. between standing up and sitting postures or between standing up and walking postures).

Posture detection evaluation is then performed by computing a DTW (i.e. Dynamic Time Warping) based metric. In order to compare the precision of the APD posturogram in regards to the ground truth, this metric measures similarity between the two temporal sequences. Table I presents our evaluation with 3 configurations: firstly, with no attitude estimation before our posture detector, secondly with Complementary Filter (CF) attitude estimator (see III-B), and thirdly with q-AKF attitude estimator (see III-C). An average posture detection error is computed from a 5-cross validation process. 5 validation datasets are used to optimize $\mathrm{CF}$ and q-AKF methods while 5 test datasets are used to evaluate blindly the precision of the full posture detection process (i.e. including attitude estimation performances). One repetition contains 25 sequences from random participants.

In Table I, we observe that the minimal average error is performed by the q-AKF attitude estimator and our posture detector with a score of $0.192646 \pm 0.023791$. This means that around $80.74 \%$ of the posture sequences were correctly classified. This score is better than using CF attitude estimator $(0.197152 \pm 0.0 .021079)$ or no attitude estimator $(0.216408 \pm 0.0009055)$. It reveals that using an attitude estimator is relevant for posture detection and that CF attitude estimation is less adequate in this context. Particularly, our protocol gives significance for dynamic phases, which may correspond better to q-AKF attitude estimation design. The main remaining confusions concern standing up and sitting postures and standing up and bending postures. On the contrary, the walking and lying down postures are mainly correctly classified by our specific detectors.

Table I: Average posture detection errors.

\begin{tabular}{|cc|c|c|}
\hline Methods & & Validation & Test \\
\hline No attitude & Mean & 0.210572 & 0.216408 \\
estimation & Standard Deviation & 0.005730 & 0.009055 \\
\hline (CF) [9] & Mean & 0.197685 & 0.197152 \\
& Standard Deviation & 0.023632 & 0.021079 \\
\hline (q-AKF) [10] & Mean & 0.190133 & $\mathbf{0 . 1 9 2 6 4 6}$ \\
& Standard Deviation & 0.025875 & 0.023791 \\
\hline
\end{tabular}

As a complement, Fig. 10a presents the influence of the parameter $K_{p}$, representing the gain in the CF formulation, on posture detection accuracy. The average minimal error over the 5 validation datasets is $0.197685 \pm 0.023632$ obtained for $K_{p}=0.8$. Likewise, Fig. 10b shows the posture detection accuracy when the parameter $P$ varies over the 5 validation datasets and the mean minimal reached error is $0.190133 \pm 0.025875$ obtained for $P=4 \times 10^{-5}$. $P$ is the initial values of the covariance matrix used in the $\mathrm{q}-\mathrm{AKF}$ attitude estimator.

\section{Conclusions And Perspectives}

We investigate the influence of two attitude estimation methods for posture detection in the particular context of everyday indoor situations. We propose an experiment based on 9 participants for evaluating the precision of posture detection. Our experiments shed light on the relative impacts of the attitude estimation computation on inertial data based posture detection. The best overall result obtains 0.192646 of errors, which is very promising to feature human behavior when performing activities of daily living.

Some challenges remain opened with large scaled datasets and environment perturbations as biases or acceleration noises due to means of transports in outdoor situations, for instance.

\section{REFERENCES}

[1] Quach Kim Anh. Extraction de caracteristiques de lactivite ambulatoire du patient par fusion dinformations de centrales inertielles. Theses, Université Lyon I, May 2012.

[2] Marc Berenguer, Marie-Jeanne Bouzid, Aida Makni, Grégoire Lefebvre, and Norbert Noury. Evolution of activities of daily living using inertia measurements: The lunch and dinner activities. Journal of the International Society for Telemedicine and eHealth, 5(0):10-1-5), 2017.

[3] Bernard Boulay. Human Posture Recognition for Behaviour. Theses, Université Nice Sophia Antipolis, January 2007. 


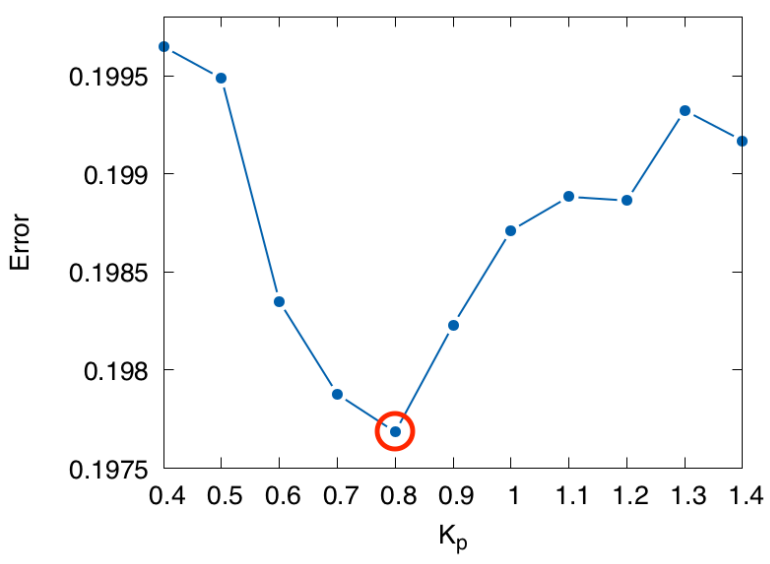

(a) Minimal error for $K_{p}$ values using (CF).

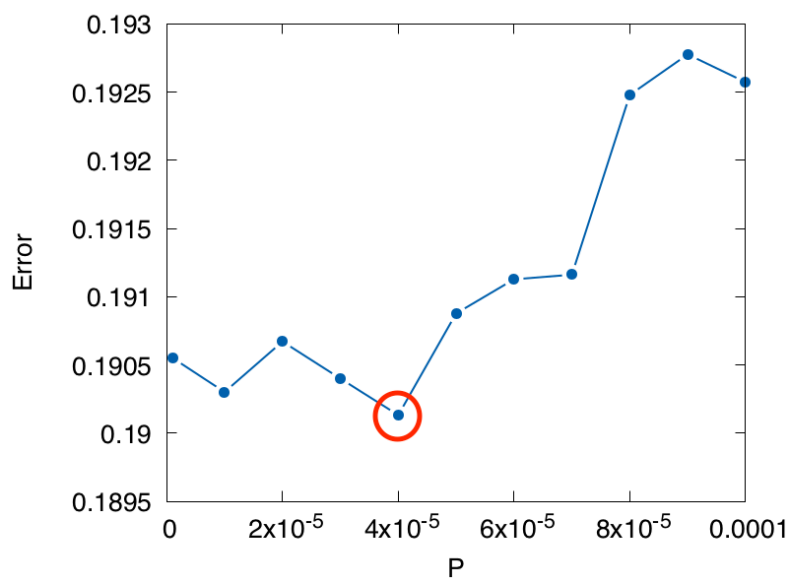

(b) Minimal error for $P$ values using (q-AKF).

Figure 10: Optimal parameters on the validation dataset.

[4] M. Euston, P. Coote, R. Mahony, J. Kim, and T. Hamel. A complementary filter for attitude estimation of a fixedwing uav. In 2008 IEEE/RSJ International Conference on Intelligent Robots and Systems, pages 340-345, Sept 2008.

[5] M. D. Hua, G. Ducard, T. Hamel, R. Mahony, and K. Rudin. Implementation of a nonlinear attitude estimator for aerial robotic vehicles. IEEE Transactions on Control Systems Technology, 22(1):201-213, Jan 2014.

[6] Wolfgang Hürst and Casper van Wezel. Gesture-based interaction via finger tracking for mobile augmented reality. Multimedia Tools and Applications, 62(1):233-258, Jan 2013.

[7] YeongJu Lee and M. Song. Selective device activation for power reduction in accelerometer-based wearable guidance systems for the blind. In 2012 IEEE International Conference on Consumer Electronics (ICCE), pages 106-107, Jan 2012.

[8] E. J. Lefferts, F. L. Markley, and M. D. Shuster. Kalman filtering for spacecraft attitude estimation. Journal of Guidance, Control, and Dynamics, 5(5):417-429, 2017/07/06 1982.

[9] Robert Mahony, Tarek Hamel, and Jean-Michel Pflimlin. Nonlinear Complementary Filters on the Special Orthogonal Group. IEEE Transactions on Automatic Control, 53(5):1203-1217, June 2008. 16.

[10] Aida Makni, Hassen Fourati, and Alain Y. Kibangou. Energyaware Adaptive Attitude Estimation Under External Acceleration for Pedestrian Navigation. IEEE/ASME Transactions on Mechatronics, January 2016.

[11] A. Moeini and M. Namvar. Global estimation of rigid-body attitude/position using a single landmark and biased velocity measurements. In 53rd IEEE Conference on Decision and Control, pages 667-672, Dec 2014.

[12] T. W. H. Nelson, L. Kam-Yiu, N. K. Y. Joseph, H. Song, and P. Ioannis. Tracking indoor activities of patients with mild cognitive impairment using motion sensors. In 2017 IEEE 31 st International Conference on Advanced Information Networking and Applications (AINA), pages 431-438, March 2017.
[13] L. Ojeda and J. Borenstein. Flexnav: fuzzy logic expert rulebased position estimation for mobile robots on rugged terrain. In Proceedings 2002 IEEE International Conference on Robotics and Automation (Cat. No.02CH37292), volume 1, pages 317-322 vol.1, 2002.

[14] Timo Pylvänäinen. Accelerometer Based Gesture Recognition Using Continuous HMMs, pages 639-646. Springer Berlin Heidelberg, Berlin, Heidelberg, 2005.

[15] Valérie Renaudin and Christophe Combettes. Magnetic, Acceleration Fields and Gyroscope Quaternion (MAGYQ) Based Attitude Estimation with Smartphone Sensors for Indoor Pedestrian Navigation. Sensors, 14(12):pp.2286422890, January 2014.

[16] M. D. Shuster. Survey of attitude representations. Journal of the Astronautical Sciences, 41:439-517, October 1993.

[17] J. F. Vasconcelos, B. Cardeira, C. Silvestre, P. Oliveira, and P. Batista. Discrete-time complementary filters for attitude and position estimation: Design, analysis and experimental validation. IEEE Transactions on Control Systems Technology, 19(1):181-198, Jan 2011.

[18] Grace Wahba. A least squares estimate of satellite attitude. SIAM Review, 7(3):409-409, 1965.

[19] John Williamson, Roderick Murray-Smith, and Stephen Hughes. Shoogle: Excitatory multimodal interaction on mobile devices. In Proceedings of the SIGCHI Conference on Human Factors in Computing Systems, CHI '07, pages 121-124, New York, NY, USA, 2007. ACM.

[20] H. Yoon, S. Hwang, D. Jung, S. Choi, K. Joo, J. Choi, Y. Lee, D. U. Jeong, and K. Park. Estimation of sleep posture using a patch-type accelerometer based device. In 2015 37th Annual International Conference of the IEEE Engineering in Medicine and Biology Society (EMBC), pages 4942-4945, Aug 2015. 